\title{
A Double Life of Freeze-Dried Enterococcus Faecium in Pet Food Industry
}

\author{
Jie $\mathrm{Xu}^{1 *}$ and Jinxia Song ${ }^{2}$ \\ ${ }^{1}$ Department of Food Science and Technology, Ohio State University, USA \\ ${ }^{2}$ Hospital of Qingdao University, China
}

*Corresponding author: Jie Xu, Department of Food Science and Technology, The Ohio State University, Columbus, OH, 43210, USA.

To Cite This Article: : Jie Xu.A Double Life of Freeze-Dried Enterococcus Faecium in Pet Food Industry. Am J Biomed Sci \& Res. 2019 - 5(6). AJBSR. MS.ID.000972. DOI: 10.34297/AJBSR.2019.05.000972.

Received: 眥 October 17, 2019; Published: 眥 October 23, 2019

\begin{abstract}
Freeze-dried Enterococcus faecium may able to lead a double life in pet food industry. Freeze-dried Enterococcus faecium can be identified both as a substitute for Salmonella in thermal processing validations and a special form of probiotics in formulations of pet foods. For the first life, freezedried Enterococcus faecium can be used as an indicator since its high thermal tolerance during processing, which is able to validate the inactivation efficiency of thermal processing in pet food industry, such as extrusion cooking. For a second life, freeze-dried Enterococcus faecium can be used as a special form of probiotics and added at the beginning of the process maintaining considerable number of variable cells, or also, can be directly added into pet foods without increasing the moisture of final products. It can be asserted that the double life of freeze-dried Enterococcus faecium satisfied both quality and nutrition aspects concerned in pet food industry.
\end{abstract}

Keywords: Enterococcus Faecium; Freeze-Dried; Validation; Probiotic; Pet Foods

\section{Introduction}

Enterococcus faecium shows ability to withstand extreme environmental conditions such as heat and salinity levels and antibiotic resistant. Reports have shown that Enterococcus faecium can be used as a surrogate for Salmonella during thermal processing, such as almond process validation [1], radio-frequency pasteurization [2-4], which can help to validate their inactivation efficiency to improve the safety condition of thermally processed foods.

Enterococcus faecium is a species of bacteria that naturally constitutes a major population in the gut as part of the normal gastrointestinal microbial flora in animals and humans. Enterococcus faecium has been used as a probiotic in some pet foods, which provides as a digestive aid and health benefits by increasing the number of healthy bacteria in the stomach [5-8]. In the Dog and Cat Food Ingredient Center database, at least 26 dry dog foods list Enterococcus faecium on their ingredient decks, including products from Orijen, Acana and Taste of the Wild [9].

Freeze-dried Enterococcus faecium has high thermal resistance, low-moisture content, long shelf-life, which has the potential to become both as a surrogate for process validation and probiotics addictive for pet foods. In this paper, a description of the double life of freeze-dried Enterococcus faecium in pet food industry is present.

\section{Freeze-Drying Technology}

Freeze-drying is currently an industrial standard for preservation of microorganisms [10,11]. However, freeze-drying can cause cells death and injury because of the changes in the physical state of the membrane lipids and protein damage or protein denaturation. However, injured cells retain their viability and upon resuscitation under a nutritionally adequate environment [12]. Factors associated with freeze-drying process (freezing speed, temperature, protectants, etc.), rehydration process (rehydration medium, temperature, $\mathrm{PH}$, etc.), and storage conditions (chances to touch moisture, oxygen, and storage temperature) can influence the survival of bacteria after freeze-drying $[13,14]$.

Protective agents during freeze-drying can be used to reduce the number of dead cells, which can protect bacterial cells during the freeze-drying process and further storage. Many studies have found that protectants can prevent fusion and thermotropic phase transitions of the cell membrane caused by freezing via electrostatic repulsion or steric hindrance [15]. Substances such as sugars, 
polymers, polyols and amino acids have been investigated for their protective effect during freeze-drying (Abadias, et al. 2001). [16] found that skim milk serves as a good protectant for Enterococcus faecium during the freeze-drying process. When the bacteria were freeze-dried with skim milk, it survived and remained heat resistant longer than bacteria protected by a buffer specifically meant for freeze-drying.

\section{Freeze-Dried Enterococcus faecium as a surrogate}

About $95 \%$ of dry pet foods are produced by extrusion cooking technology [17]. There are multiple numbers of outbreaks of salmonellosis have been associated with extruded pet foods $[18,19]$. In this case, the inactivation efficiency of extrusion technology is needed to be validated by using a nonpathogenic, heat resistance, surrogate microorganism, such as Enterococcus faecium. Several studies have reported the ability of using Enterococcus faecium as a surrogate of Salmonella to validate extrusion processing [2022]. found that Enterococcus faecium was inactivated at higher temperatures than Salmonella, indicating that its use as a surrogate would provide an appropriate margin of error in extrusion processes designed to eliminate this pathogen. The ability of using freeze-dried Enterococcus faecium as a surrogate for validation was firstly reported by Xu et al. Xu found that freeze-dried Enterococcus faecium has a comparable thermal resistance with its liquid form and can be used as an indicator for radio-frequency pasteurization validation of wheat flour [23].

\section{Freeze-Dried Enterococcus faecium as probiotics}

Freeze-dried Enterococcus faecium can survive better during extrusion process since its high thermal tolerance. Extrusion cooking is a process with thermomechanical treatment involving high-temperature conditions as high as $135^{\circ} \mathrm{C}$ [24]. If Enterococcus faecium is included in the pet food or treat recipe before extrusion, the probiotic could be killed by the heat, or reduced in effectiveness [9]. Freeze-dried Enterococcus faecium with protectant showed a fairly good resistance to heat, which provided a more heat resistant way of including the bacteria could improve the probiotic quality of the final products $[9,23]$.

Freeze-dried Enterococcus faecium can be used as a special form of probiotics without introducing extra moisture to the final products. The introduction of liquid inoculants can change the physical characteristics of foods and requires extra waiting while the product returns to its intended moisture level. By using dryform probiotic, the changes in food properties can be avoided and the need of extra time for re-equilibration can be eliminated.

Freeze-dried Enterococcus faecium is storage stable and remain viable during long time storage [23]. The cell counts for many probiotic microorganisms may fall away completely within one or two days, resulting a big issue incorporating probiotic microorganisms into foodstuffs [25]. Developed a ready-to-eat cereal product includes a coating or filling containing a probiotic micro-organism. The probiotic microorganism was spray-dried with a suitable carrier substrate and has remain viable during long time storage. Compared with coating method, freeze-dried microbes are shelf stable, and faster and easier to prepare.

\section{Conclusions}

Freeze-dried Enterococcus faecium, with a high tolerant to thermal treatment, can be used as a surrogate of Salmonella for processing validation in pet industry, or, as a special form probiotics of pet foods added at the beginning of the process or directly added into final products. However, the functionality of Enterococcus faecium after freeze-drying has to be further confirmed. Further systematic studies are needed for its application in pet food area.

\section{Acknowledgements}

We acknowledge the support from Dr. Juming Tang, regent professor of Department of Biological Systems Engineering in Washington State University, for providing all the facilities and financial supports on author Jie Xu's studies on freeze-dried Enterococcus faecium. Special appreciation for Tim Wall, a reporter from PetfoodIndustry.com is also acknowledged.

\section{Conflict of interest}

There is no conflict of interest among Authors and Co-authors and have no conflict of interest to declare.

\section{References}

1. ABC (2007) "Almond Board of California. Guidelines for Process Validation Using Enterococcus Faecium NRRL B-2354".

2. Vill Rojas R (2015) Influence of different factors on desiccation survival and thermal resistance of Salmonella and radiofrequency pasteurization of low-moisture foods. Washington State University.

3. Shuxiang Liu, Samet Ozturk, Jie Xu, Fanbing Kong, Peter Gray, et al. (2017). "Microbial validation of radio frequency pasteurization of wheat flour by inoculated pack studies." Journal of Food Engineering p. 68-74.

4. Wei X (2017) "Radiofrequency processing for inactivation of Salmonella spp. and Enterococcus faecium NRRL B-2354 in whole black peppercorn and ground black pepper." University of Nebraska-Lincoln.

5. Benyacoub J, Czarnecki Maulden GL, Cavadini C, Sauthier T, Anderson RE, et al. (2003) "Supplementation of food with Enterococcus faecium (SF68) stimulates immune functions in young dogs." The Journal of nutrition 133(4): 1158-1162.

6. Marciňáková M, Simonová M, Strompfová V, Lauková A (2006) “Oral application ofEnterococcus faecium strain EE3 in healthy dogs". Folia microbiologica 51(3): 239-242.

7. Zink R, et al. (2007) Probiotics for pet food applications, Google Patents.

8. Petnet (2014) "Enterococcus Faecium: Pet Food Ingredients A to Z".

9. Wall T (2017) "Probiotic freeze-dried microbes may help pet food safety." Using freeze-dried E. faecium could streamline kill step validation and boost probiotic survival.

10. Bjerketorp J, Håkansson S, Belkin S, Jansson JK (2006). "Advances in preservation methods: keeping biosensor microorganisms alive and active." Current opinion in biotechnology 17(1): 43-49. 
11. Morgan CA, Herman N, White PA, Vesey G (2006) "Preservation of microorganisms by drying; a review." Journal of Microbiological Methods 66(2): 183-193.

12. Bretz HW, FE Kocka (1967) "Resistance to actinomycin D of Escherichia coli after frozen storage." Canadian Journal of Microbiology 13(7): 914 917.

13. Sinskey T, G Silverman (1970) "Characterization of injury incurred by Escherichia coli upon freeze-drying." Journal of Bacteriology 101(2): 429-437.

14. Miyamoto Shinohara Y, Sukenobe J, Imaizumi T, Nakahara T (2008) "Survival of freeze-dried bacteria." The Journal of general and applied microbiology 54(1): 9-24.

15. Berner D, H Viernstein (2006) "Effect of protective agents on the viability of Lactococcus lactis subjected to freeze-thawing and freeze-drying." Scientia Pharmaceutica 74(3): 137-149.

16. Jie Xu, Shuxiang Liu, Jinxia Song, Juming Tang, Mei-Jun Zhu, et al. (2017) Dry-inoculation method for thermal inactivation studies in wheat flour using freeze-dried Enterococcus faecium NRRL B-2354. LWT-Food Science and Technology 89: 10-17.

17. Spears JK, GC Fahey (2004) "Resistant starch as related to companion animal nutrition." Journal of AOAC International 87(3): 787-791.

18. Centers for Disease Control and Prevention (2008) “Multistate Outbreak of Human Salmonella Infections Caused by Contaminated Dry Dog Food --United States, 2006--2007." MMWR Morb Mortal Wkly Rep 57(19): 521-524.
19. Centers for Disease Control and Prevention (2012) "Multistate Outbreak of Human Salmonella Infantis Infections Linked to Dry Dog Food (Final Update)".

20. Bianchini A, Stratton J, Weier S, Hartter T, Plattner B, et al. (2012) "Validation of extrusion as a killing step for Enterococcus faecium in a balanced carbohydrate-protein meal by using a response surface design." Journal of Food Protection 75(9): 1646-1653.

21. Bianchini A, Stratton J, Weier S, Hartter T, Plattner B, et al. (2014) "Use of Enterococcus faecium as a surrogate for Salmonella enterica during extrusion of a balanced carbohydrate-protein meal." Journal of Food Protection 77(1): 75-82.

22. Verma T, Wei X, Lau SK, Bianchini A, Eskridge KM, et al. (2018) "Evaluation of Enterococcus faecium NRRL B-2354 as a surrogate for Salmonella during extrusion of low-moisture food." Journal of Food Science 83(4): 1063-1072.

23. Jie Xu, Shuxiang Liu, Juming Tang, Samet Ozturk, Fanbin Kong, et al. (2018) "Application of freeze-dried Enterococcus faecium NRRL B-2354 in radio-frequency pasteurization of wheat flour." LWT-Food Science and Technology 90: 124-131.

24. Coelho M (2002) Vitamin stability in premixes and feeds: A practical approach in ruminant diets. Proceedings 13th Annual Florida Ruminant Nutrition Symposium pp. 127-154.

25. Christof Cavadini OB, Walter Gaier (1999) Pet food product containing probiotics. Google Patents US5968569A. 